1. Greinacher A, Friesecke S, Abel P, et al. Treatment of severe neurological deficits with IgG depletion through immunoadsorption in patients with Escherichia coli O104:H4-associated haemolytic uraemic syndrome: a prospective trial. Lancet 2011; 378:1166-73.
2. Cornec-Le Gall E, Delmas Y, De Parscau L, et al. Adult-onset eculizumab-resistant hemolytic uremic syndrome associated with cobalamin C deficiency. Am J Kidney Dis 2014;63:119-23.

\title{
Case 25-2014: A Man with Ulcerative Colitis and Bloody Diarrhea
}

TO THE EDITOR: The Case Record contributed by Hohmann et al. (Aug. 14 issue), ${ }^{1}$ regarding a man with ulcerative colitis in whom bloody diarrhea developed soon after fecal microbiota transplantation (FMT), highlights the difficulty in distinguishing cytomegalovirus (CMV) as a direct cause of disease from its being an "innocent bystander" in patients with CMV infection and active ulcerative colitis. However, the authors also reported an apparent case of diarrheal disease caused by Blastocystis hominis transmitted by means of FMT without describing the complex debate as to whether this organism is a true enteropathogen.

In developed countries, blastocystis is detectable in more than $50 \%$ of healthy persons with the use of polymerase-chain-reaction-based assays. ${ }^{2}$ No difference in the prevalence of blastocystis exists between hosts with gastrointestinal symptoms and those without such symptoms, ${ }^{3}$ and symptoms that are attributed to blastocystis infection may not improve even after the elimination of the organism. ${ }^{4}$ The organism has been shown to be capable of both long-term colonization $^{2}$ and spontaneous disappearance without intervention. ${ }^{4,5}$ Physicians offering FMT should carefully monitor FMT recipients for infectious complications but must be aware that presence is not the same as causation.

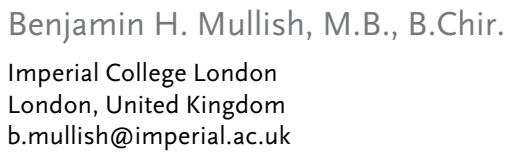

No potential conflict of interest relevant to this letter was reported.

1. Case Records of the Massachusetts General Hospital (Case 25-2014). N Engl J Med 2014;371:668-75.

2. Scanlan PD, Stensvold CR, Rajilić-Stojanović M, et al. The microbial eukaryote Blastocystis is a prevalent and diverse mem- ber of the healthy human gut microbiota. FEMS Microbiol Ecol 2014 July 30 (Epub ahead of print).

3. Udkow MP, Markell EK. Blastocystis hominis: prevalence in asymptomatic versus symptomatic hosts. J Infect Dis 1993;168: 242-4.

4. Sun T, Katz S, Tanenbaum B, Schenone C. Questionable clinical significance of Blastocystis hominis infection. Am J Gastroenterol 1989;84:1543-7.

5. Grossman I, Weiss LM, Simon D, Tanowitz HB, Wittner M. Blastocystis hominis in hospital employees. Am J Gastroenterol 1992;87:729-32.

DOI: $10.1056 /$ NEJMc1410949

THE DISCUSSANT REPLIES: We understand the controversy surrounding $B$. hominis (an anaerobic protozoan), and the case referenced in the discussion, reported by a Canadian physician, highlights it nicely. The patient had recurrent Clostridium difficile infection, and the spousal donor, who had no gastrointestinal symptoms, had stool that tested positive for $B$. hominis (no other donor was readily available). The FMT cured the $C$. difficile infection, but intermittent loose stools lasting months subsequently developed, with positive tests for blastocystis. A course of nitazoxanide cured the symptoms.

At a recent American Gastroenterological Association conference on FMT, physicians noted that blastocystis was a common finding in donor stool specimens (e.g., in 6 of 38 specimens that were screened in an Australian study). ${ }^{1}$ The consensus of practitioners was that it would not be advisable to use such specimens for FMT. Attendees did not mention other cases of transmission of infectious agents by FMT. Representatives of the Food and Drug Administration highlighted the possibility of transmission of currently unknown infectious agents and the need for judicious use and careful follow-up of recipients of FMT. 


\section{Elizabeth L. Hohmann, M.D.}

Massachusetts General Hospital

Boston, MA

ehohmann@partners.org

Since publication of her article, the author reports no further potential conflict of interest.
1. Paramsothy S, Borody T, Lin E, et al. Obstacles to donor recruitment for fecal microbiota transplantation: experiences from the FOCUS Study. Presented at Therapeutic Innovations in Microbiome Research and Technology AGA Freston Conference, Chicago, August 13-14, 2014.

DOI: 10.1056/NEJMc1410949

\section{Catheterization of the Urethra in Girls}

TO THE EDITOR: As Manzano et al. noted in their Video in Clinical Medicine (July 10 issue), ${ }^{1}$ it is sometimes difficult for the examiner to identify the urethral meatus when attempting to perform catheterization of the urinary bladder in girls. In such instances, it may be helpful to draw the labial tissue upward by moving the nondominant hand anteriorly after spreading the labia majora with two fingers. ${ }^{2}$

If the meatus remains hard to visualize, we suggest enlisting an assistant for the following maneuver, which we term "labial lift." Both the examiner and the assistant should gently grasp one of the labia majora - the examiner on the side of her nondominant hand and the assistant opposite. Together, they lift the labia anteriorly, laterally, and caudad (Fig. 1). As shown in the figure, using the corner of a small piece of sterile gauze helps to maintain a comfortable hold

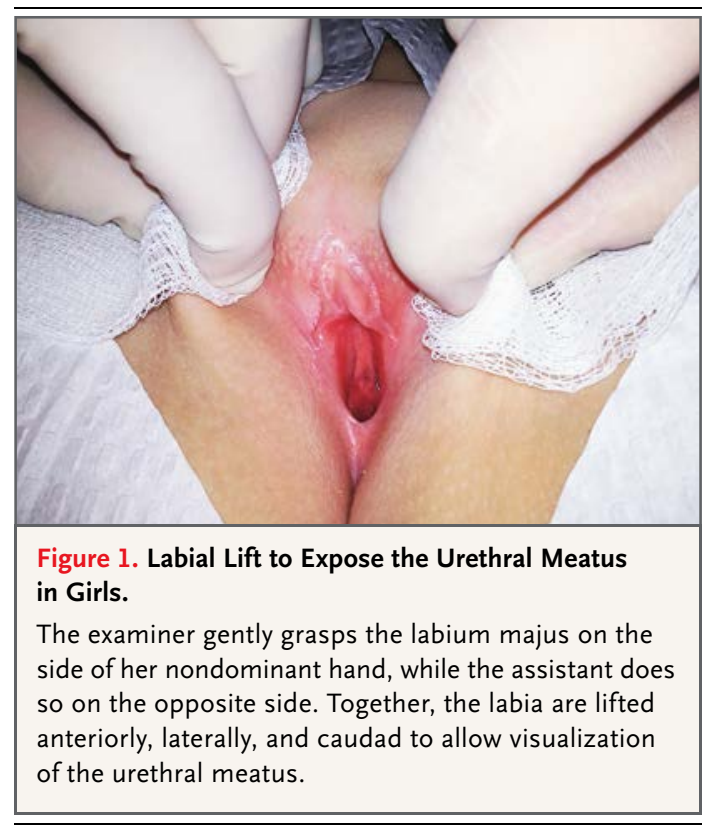

on the labia, which may become slippery after sterile preparation. We have found that this technique seldom fails to allow the necessary visualization of the meatus.

Mark A. Faasse, M.D., M.P.H.

Max Maizels, M.D.

Ann and Robert H. Lurie Children's Hospital of Chicago

Chicago, IL

mfaasse@luriechildrens.org

No potential conflict of interest relevant to this letter was reported.

1. Manzano S, Vunda A, Schneider F, Vandertuin L, Lacroix LE. Videos in clinical medicine: catheterization of the urethra in girls. N Engl J Med 2014;371(2):e2.

2. Cartwright PC, Wallis MC. Office procedures. In: Palmer JS, ed. Current clinical urology: pediatric urology. New York: Humana Press, 2011:127-40.

DOI: 10.1056/NEJMc1410733

THE AUTHORS REPLY: This is a very nice alternative to the use of downward traction to the ceph-

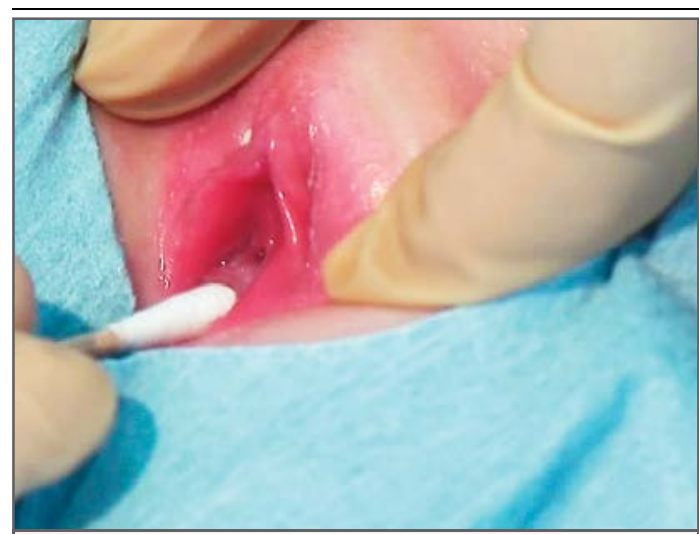

Figure 1. Downward Traction of the Vaginal Mucosa.

The examiner localizes the meatus by gently pulling the cephalad fold of the vaginal introitus downward. 